\title{
Mucoepidermoid carcinoma of the anal canal: A case report and review of the literature
}

\author{
MANABU YAMAMOTO ${ }^{1}$, KEIJI HIRATA ${ }^{2}$, MASAZUMI TUNEYOSHI ${ }^{3}$, YOSHIHIRO YOSHIDA ${ }^{1}$, \\ HIROMITSU MATSUDA ${ }^{1}$, TOMONOBU GION ${ }^{1}$ and YOHEI TOMINAGA ${ }^{1}$ \\ ${ }^{1}$ Department of Surgery, Fukuoka Sanno Hospital, Fukuoka, Fukuoka 814-0001; \\ ${ }^{2}$ Department of Surgery I, School of Medicine, University of Occupational and Environmental Health, Kitakyushu, \\ Fukuoka 807-8555; ${ }^{3}$ Department of Pathology, Fukuoka Sanno Hospital, Fukuoka, Fukuoka 814-0001, Japan
}

Received October 23, 2017; Accepted August 1, 2018

DOI: $10.3892 /$ mco.2018.1706

\begin{abstract}
Mucoepidermoid carcinoma of the anal canal is a rare tumor. We herein report the case of a 74-year-old male patient with a high-grade mucoepidermoid carcinoma of the anal canal who was treated by local surgical resection and subsequent irradiation. However, the patient succumbed to liver and lung metastases 2 years after the procedure. The characteristic findings of mucoepidermoid carcinoma of the anus remain unclear to date due to rarity of this tumor. Since 1954, when this type of tumor was first described, only 58 cases of patients diagnosed with mucoepidermoid carcinoma of the anus have been reported to date. In this context, a review of the existing English literature on this rare tumor was also performed.
\end{abstract}

\section{Introduction}

Primary malignant tumors of the anus are histologically diverse and account for only $2 \%$ of all malignancies of the large bowel (1-3). Mucoepidermoid carcinoma of the anal canal, a type of primary malignant tumor, is a rare lesion that is characterized by goblet cell-type mucous cells arranged in solid sheets. To date, only 58 cases have been reported in the literature (4-17). The origin of this tumor is a subject of debate, and its precise histogenesis also remains unclear. We herein present the case of a 74-year-old man with a mucoepidermoid carcinoma of the anal canal.

\section{Case report}

A 74-year-old Japanese male patient was admitted to the Fukuoka Sanno Hospital (Fukuoka, Japan) in January 2013

Correspondence to: Dr Manabu Yamamoto, Department of Surgery, Fukuoka Sanno Hospital, Momochi-hama 3-6-45, Sawara-ku, Fukuoka, Fukuoka 814-0001, Japan

E-mail: myamamoto@kouhoukai.or.jp

Key words: anal, mucoepidermoid, carcinoma with complaints of anal pain. Anorexia and vomiting were not reported. Measurement of serum carcinoembryonic antigen and carbohydrate antigen 19-9 revealed that they were below the cut-off levels. Endoscopy revealed a Bormann type II tumor lesion $(25 \times 20 \mathrm{~mm})$ in the anal canal (Fig. 1), and biopsy revealed an adenocarcinoma with a squamous cell carcinoma component. Computed tomography revealed enlarged lymph nodes in the right inguinal region and around the right external iliac artery. Resection of the tumor and excisional biopsy of the right inguinal lymph nodes were performed on January 25 , 2013, as the patient requested minimally invasive surgery. The patient was discharged on the second postoperative day. The resected specimen was analyzed under an optical microscope at the Armed Forced Institute of Pathology, and pathological examination revealed a high-grade mucoepidermoid carcinoma (Fig. 2A and B), and immunohistochemical staining by p40 and alcian blue $(4 \mu \mathrm{m})$. The blue color indicates the mucin production stained by alcian blue (room temperature, $70 \mathrm{~min}$ ), and the brown color represents the squamoid cells stained by $\mathrm{p} 40$ (room temperature $-98^{\circ} \mathrm{C}, 180 \mathrm{~min}$ ) (Fig. $2 \mathrm{C}$ and $\mathrm{D}$ ), with a TNM classification of T2, N3, M0 (stage IIIB). Tumor specimens $(2 \mu \mathrm{m})$ were examined via hematoxylin and eosin (H\&E; staining at room temperature for a total of $50 \mathrm{~min}$ and analyzed under and optical microscope. The patient underwent radiotherapy in the pelvic and inguinal regions postoperatively; however, he succumbed to lung and liver metastases 2 years postoperatively.

\section{Discussion}

Carcinoma of the anal canal is a comparatively rare disease, constituting only $2 \%$ of all malignancies of the large bowel, including the anus (1-3). Some investigators have reported that 4-6.9\% of anal carcinomas are diagnosed as mucoepidermoid carcinomas $(10,15)$. However, mucoepidermoid carcinoma of the anus is considered to be a rare lesion. This type of tumor was first described by Kay in 1954, and a total of 58 cases have been reported to date (4-17).

The histogenesis of this tumor has been discussed in the literature. Kay reported that mucoepidermoid carcinoma of the anus arises from 6-8 anal glands, the ducts of which drain into the anal crypts. Fenger and Filip compared the staining 


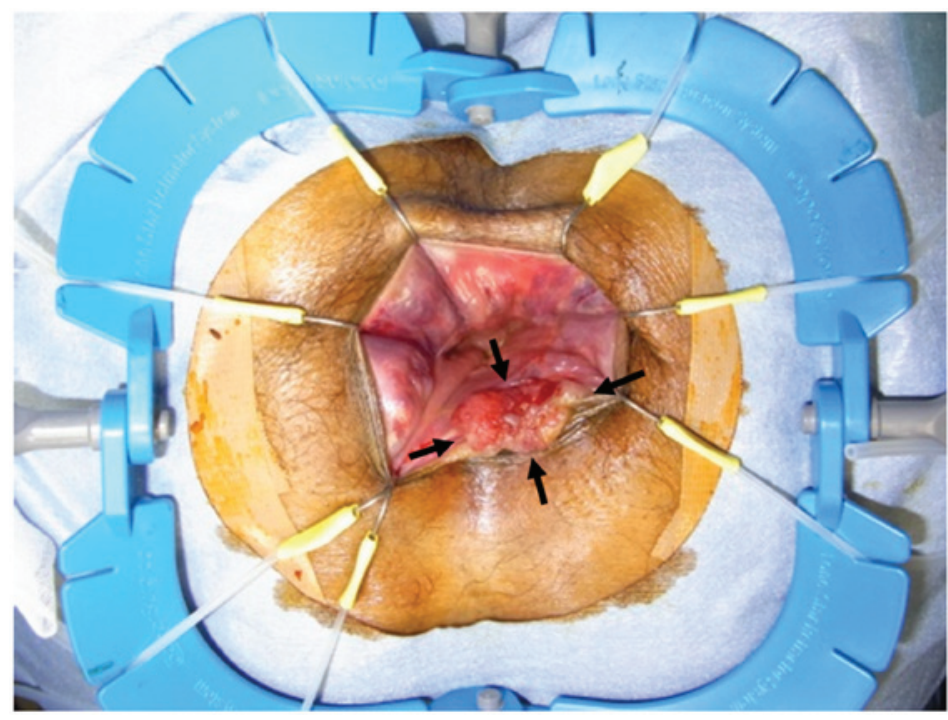

Figure 1. Intraoperatively, a Bormann type II tumor lesion (25x20 mm, indicated by arrows) was identified in the anus.
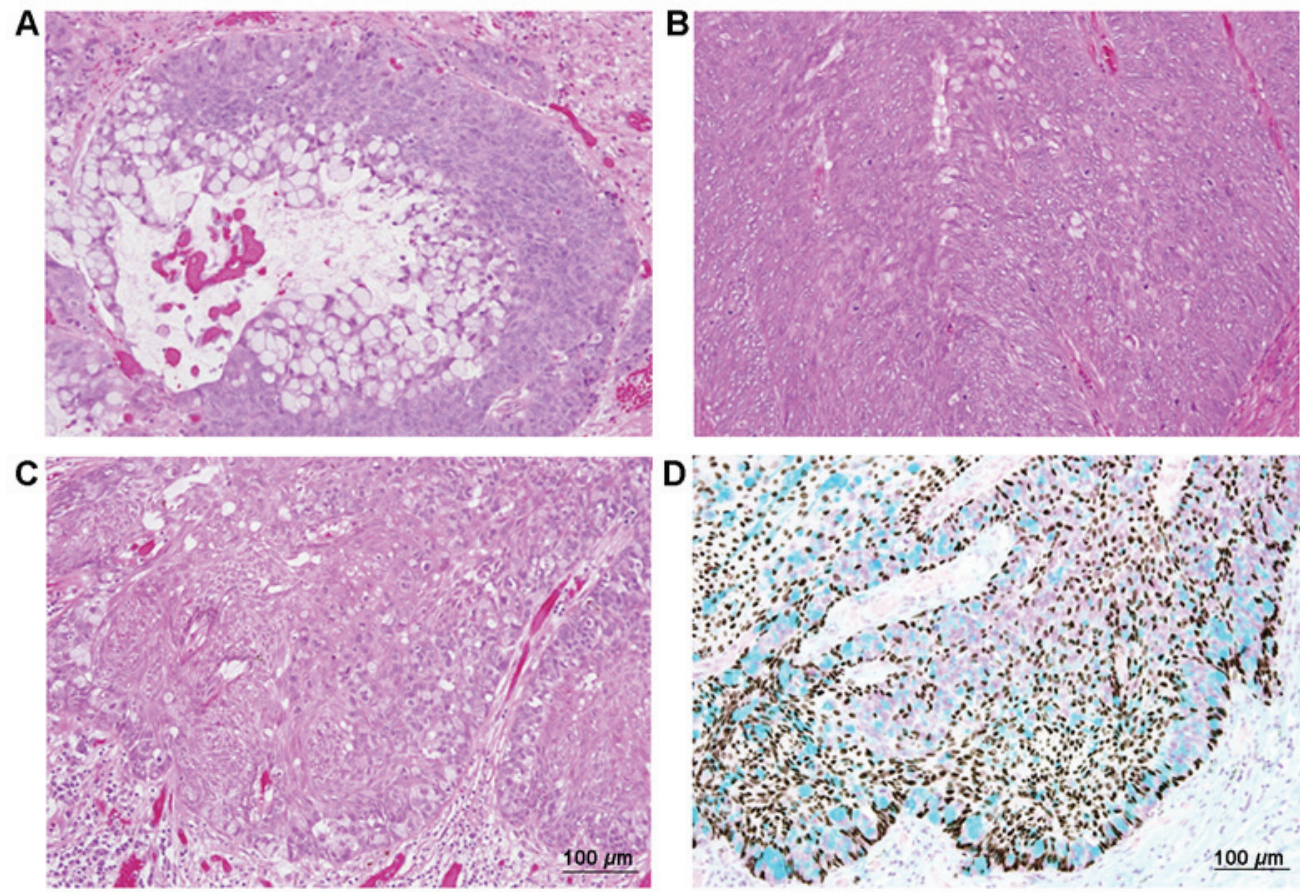

Figure 2. Hematoxylin and eosin (H\&E) staining of the tumor specimen revealed the presence of four cell types, namely mucin-producing, squamous, intermediate and clear cells. In the present case, the squamous component was predominant ( $2 \mu \mathrm{m}$, room temperature, 50 min). There was no lymphatic or vascular invasion identified in the tumor. (A) Mucoepidermoid (magnification, $\mathrm{x} 100$ ) and (B) squamous cell carcinoma components (magnification, $\mathrm{x} 100)$. (C) H\&E staining in same speciment for D lesion (magnification, $\mathrm{x} 100$ ). (D) Immunohistochemical staining by $\mathrm{p} 40$ and alcian blue ( $4 \mu \mathrm{m}$, room temperature $-98^{\circ} \mathrm{C}$, $180 \mathrm{~min}$ and room temperature, $70 \mathrm{~min}$ ). The blue color indicates the mucin production stained by alcian blue, and the brown color represents the squamoid cells stained by p40 (magnification, x100).

characteristics of the mucous component of a mucoepidermoid carcinoma with those of the rectal mucosa and anal glands, and concluded that the staining patterns of mucoepidermoid carcinoma were similar to those of the anal glands (9). By contrast, Morson and Volkstadt and Fenger and Nielsen demonstrated by morphological examination that mucoepidermoid carcinomas arise from the transitional zone, similar to the basaloid and certain other types of squamous cell carcinoma $(7,18)$. The case reported herein falls into the latter category.

Data from 35 cases of mucoepidermoid carcinoma were summarized, including details such as age, sex, treatment and prognosis (Table I). The mean age \pm standard deviation was $55.7 \pm 11.3$ years, and the male:female ratio was 0.94 . All 35 patients had undergone surgery, radiation, and/or chemotherapy. However, the majority of the patients had undergone abdominoperineal resection. By contrast, Papillon and Chassard reported that mucoepidermoid T4 tumors, similar to the epidermoid carcinomas of the anal margin, were suitable for preoperative irradiation and delayed surgery (14). Moreover, the treatment regimens were adjusted according to the depth of tumor invasion or tumor size. The 5 -year survival rate in the 35 cases summarized 
Table I. Characteristic findings in 35 patients with mucoepidermoid carcinoma.

\begin{tabular}{lc}
\hline Characteristics & Values \\
\hline Age (years) & $55.7 \pm 7.3$ \\
Sex & \\
Male:female & $17: 18$ \\
Tumor size $(\mathrm{cm})^{\mathrm{a}}$ & $4.09 \pm 2.44$ \\
Treatment & \\
APR & $22(62.9 \%)$ \\
Local resection & 2 \\
APR + RT & 4 \\
APR + chemotherapy & 1 \\
Local resection + RT & 2 \\
RT & 4 \\
Prognosis (survival for $>5$ years) & $11(31.4 \%)$ \\
\hline
\end{tabular}

Values are presented as mean \pm standard deviation, no. (\%), or as absolute numbers. ${ }^{\text {DD }}$ ata reported from 12 cases. APR, abdominoperineal resection; RT, radiation therapy.

in the present study was $31.4 \%$, and all the patients had a poor prognosis. Although there have been no reports on the prognosis of mucoepidermoid carcinoma, studies on the prognosis of epidermoid carcinoma reported 5-year survival rates as low as 26 and $32 \%(8,14)$.

In conclusion, we herein presented the case of a patient with mucoepidermoid carcinoma of the anus and discussed treatment and prognosis based on previous reports of 35 similar cases. An abdominoperitoneal resection is usually performed in established cases of mucoepidmoid carcinoma of the anus. The prognosis is generally considered to be poorer compared with that of the more common rectal carcinoma.

\section{Acknowledgements}

The authors would like to thank Mr. Fuminori Sakanashi (Department of Pathology, Fukuoka Sanno Hospital, Fukuoka, Japan) for technical assistance with immunochemical staining.

\section{Funding}

The present study was supported by the Uehara Memorial Foundation (grant no. 201620024).

\section{Availability of data and materials}

Not applicable.

\section{Authors' contributions}

MY contributed to the design of the present study and the writing of the manuscript. KH, YY, HM, TG and YT made substantial contributions to the acquisition of data. MT contributed to the diagnosis of the mucoepidermoid carcinoma. All authors read and approved the final manuscript.

\section{Ethics approval and consent to participate}

The present study was approved by the Institutional Review Board of Fukuoka Sanno Hospital. Written informed consent was obtained from the patient's family.

\section{Patient consent for publication}

Consent for the publication of data and any associated images was obtained from the patient's family.

\section{Competing interests}

The authors declare that they have no competing interests.

\section{References}

1. Beahrs OH and Wilson SM: Carcinoma of the anus. Ann Surg 184: 422-428, 1976.

2. Golden GT and Horsley JS III: Surgical management of epidermoid carcinoma of the anus. Am J Surg 131: 275-280, 1976.

3. National Board of Health and Welfare: Cancer incidence in Sweden 1975 and 1976. In: The Cancer Registry. Liber Förlag, Stockholm, pp140-152 1980.

4. Kay S: Mucoepidermoid carcinoma of the anal canal and its relation to the anal ducts. Cancer 7: 359-366, 1954.

5. Close AS and Schwab RL: A history of the anal ducts and anal-duct carcinoma; report of a case. Cancer 8: 979-985, 1955

6. Berg JW, Lone F and Stearns MW: Mucoepidermoid anal cancer. Cancer 13: 914-916, 1960.

7. Morson BC and Volkstädt H: Muco-epidermoid tumors of the anal canal. J Clin Pathol 16: 200-205, 1963.

8. Seidenverg N and Kleinhenz RJ: Mucoepidermoid carcinoma of the anus. Am J Surg 117: 413-415, 1969.

9. Fenger C and Filip MI: Pathology of the anal glands with special references to their mucin histochemistry. Acta Pathol Microbiol Scand A 85: 273-285, 1977.

10. Bohe M, Lindström C, Ekelund G and Leandoer L: Carcinoma of the anal canal. Scand J Gastroenterol 17: 795-800, 1982.

11. Dougherty BG and Evans HL: Carcinoma of the anal canal: A study of 79 cases. Am J Clin Pathol 83: 159-164, 1985.

12. Giltman LI, Osborne PT, Coleman SA and Uthman EO: Paget's disease of the anal mucosa in association with carcinoma demonstrating mucoepidermoid features. J Surg Oncol 28: 277-280, 1985.

13. Shepherd NA, Scholefieeld JH, Love SB, England J and Northover JM: Prognostic factors in anal squamous carcinoma: A multivariate analysis of clinical, pathological and flow cytometric parameters in 235 cases. Histopathology 16: 545-555, 1990.

14. Papillon J and Chassard JL: Respective roles of radiotherapy and surgery in the management of epidermoid carcinoma of the anal margin. Series of 57 patients. Dis Colon Rectum 35: 422-429, 1992.

15. Gabriele AM, Rovea P, Sola B, Trotti AB and Comandone A: Radiation therapy and chemotherapy in the conservative treatment of carcinoma of the anal canal: Survival and late morbidity in a series of 25 patients. Anticancer Res 17 (1B): 653-656, 1997.

16. Kondo R, Hanamura N, Kobayashi M, Seki T, Adachi W and Ishii K: Mucoepidermoid carcinoma of the anal canal: An immunohistochemical study. J Gastroenterol 36: 508-514, 2001.

17. Fogler R, Lanter B, Stern G and Weiner E: Mucoepidermoid carcinoma in an 'anal fistula' with associated adenocarcinoma in a villous adenoma of the descending colon: Report of a case. Dis Colon Rectum 20: 428-435, 1977.

18. Fenger $\mathrm{C}$ and Nielsen VT: Precancerous changes in the anal canal epithelium in resection specimens. Acta Pathol Microbiol Immunol Scand A 94: 63-69, 1986. 